\title{
REFLEXIONES EN TORNO A LA ENSENAANZA DE LA HISTORIA EN COLOMBIA: UN BREVE BALANCE HISTORIOGRÁFICO
}

\section{REFLECTIONS ON THE TEACHING OF HISTORY IN COLOMBIA: A BRIEF HISTORIOGRAPHICAL BALANCE}

\author{
Juan David Restrepo Zapata*
}

RESUMEN

El artículo realiza una reflexión de la enseñanza de la historia en Colombia, a partir de la recopilación de los principales manuales utilizados por el Estado para impartir los cursos de Historia en el país. Se analiza las diferentes formas de abordar la historia a lo largo de la vida republicana colombiana, para identificar aspectos de la construcción nacional, del uso político de esta y del avance disciplinar de la historia que se ha escrito y enseñado, sin desconocer el contexto internacional que ha influenciado a Colombia. En dicho sentido, este estudio tiene como objetivo ofrecer una mirada panorámica al desarrollo y evolución de la enseñanza de la historia en el país, teniendo en cuenta los procesos de la vida nacional, las influencias educativas y políticas, así como, el significado de enseñar esta ciencia social en un país tan complejo como Colombia.

PALABRAS CLAVE: ENSEÑANZA * HISTORIA * COLOMBIA * EDUCACIÓN * HISTORIOGRAFÍA

\section{ABSTRACT}

The article makes a reflection of the teaching of history in Colombia from the compilation of the main manuals used by the State to teach History courses in the country. It is analyzed the different ways of approaching history throughout of the Colombian republican life, for identify aspects of the national construction, of the political use of this one, and of the disciplinary advance of the history that was written and taught, this without ignoring the international context from which Colombia was influenced. In this sense, this study aims to offer a panoramic view of the development and evolution of the teaching of history in the country, taking into account the processes of national life, educational and political influences and the meaning of teaching this social science in a country as complex as Colombia.

KEYWORDS: TEACHING * HISTORY * COLOMBIA * EDUCATION * HISTORIOGRAPHY

Grupo de Investigación de Estudios Interdisciplinares en Historia General, Facultad de Ciencias Sociales y Humanas, Universidad de Antioquia, Colombia.

juandrestrepozapata@gmail.com 


\section{INTRODUCCIÓN}

La historia como disciplina científica en constante construcción, merece profundas e ineludibles reflexiones en torno a su estudio, metodología, objetivos $y$, como es el caso de este escrito, de su enseñanza. La historia es heredera de unos ideales decimonónicos, donde legitimar y construir el poder de las fructíferas burguesías del viejo y nuevo continente, se hacía más que nunca inaplazable. Edificar un conjunto de discursos enfocados en el pasado, representaba una de las más valiosas herramientas para los proyectos nacionales que se alzaban en un mundo que intentaba dejar atrás el Antiguo Régimen y que veían en la exaltación de los valores liberales y republicanos, el culmen de su formación como sociedad.

Por lo tanto, en el siglo xix, se vio en la escritura de la historia un "arma" (Moreno, 1999), que defendía la construcción de los Estadosnación. Muchos de los proyectos historiográficos giraban en torno a dicha idea $y$ poco a poco contribuían a fortalecer la "comunidad imaginada" (Anderson, 1993). Algunos, desde territorio europeo, vieron en el historicismo alemán o en el positivismo rankeano unas corrientes metódicas que vivificaban el pasado con "total verdad", acudiendo al documento como verdadero transmisor de información de un tiempo oculto que esperaba ser recuperado. Humboldt $y$ Von Ranke indicaban que la historia necesitaba acudir a fuentes fidedignas, que debían pasar por una sistemática crítica, donde se demostrara su autenticidad. Ello aseguraría que fuera tangible la voz de la realidad, sin importar cuantos años mediaran entre su escritura y su interpretación (Corcuera de Mancera, 1997).

Sin embargo, las convulsiones por las que atravesaba la sociedad europea de ese entonces, donde la industrialización y el trabajo asalariado dividían a la humanidad entre "detentadores de los medios de producción y proletarios", motivaron a pensadores a asumir una posición política de lucha, que pudiera destruir el modelo capitalista y erigir una sociedad comunista. Es así que Carlos Marx con sus propuestas vivificó una historia combativa, que acudiera a un materialismo histórico para analizar y divulgar el desarrollo por estadios de la historia, algo que más tarde, aunque con algunas posturas diferentes, sentaría las bases de la historia social (Hobsbawm, 1983).

La llegada del siglo xx trajo nuevos retos a la historia. Muchos presagiaban "la decadencia de occidente" (Spengler, 2014), mientras que el enfrentamiento entre sistemas ocasionaba millones de víctimas; transformándose así la disciplina que pensaba la acción de las elites en el pasado, en una disciplina que entendía e indagaba el presente, con una conciencia del pasado. Esto se encarnó en la escuela de los Annales, que teniendo como fundadores a Lucien Febvre y Marc Bloch, cuestionó las tradicionales miradas de la historia e impulsó a la disciplina a adoptar unas visiones integrales, que tuvieran en cuenta la Economía, la Sociología, la Geografía, entre otros, produciéndose así unos verdaderos "combates por la historia" (Febvre, 1970), que devolverían a la disciplina su espíritu político.

El análisis del pasado se convirtió en una ciencia que pensaba al hombre en sociedad, con una mirada crítica, donde su interdisciplinariedad concedía nuevas miradas sobre nuevos sujetos. En la escuela de Annales, Fernand Braudel desarrolló toda una propuesta que separaba metodológicamente el estudio de la historia en tres tiempos - la larga, mediana y corta duración-, que complejizaban y prolongaban el análisis, pero que lo hacían más fuerte y enérgico (Braudel, 1974). Después, la historia de las mentalidades y las nuevas corrientes historiográficas, como el giro lingüístico, de Hayden White o la microhistoria italiana y mexicana, propusieron nuevas visiones sobre el pasado y su forma de abordarlo. Curiosamente, la caída del muro de Berlín, motivó el planteamiento de tesis fuertemente criticadas que auguraban "el fin de la Historia" (Fukuyama, 1992). Sin embargo, el análisis prolongado de todo lo anterior supera los objetivos de este escrito.

Transversalmente, la enseñanza de la historia perseguía las transformaciones disciplinares para incorporarlas a sus saberes, por lo tanto, realizar una reflexión sobre ello, implica ir al fondo de la disciplina por sí misma. La "instrucción pública" delineada desde el 
ascenso al poder de los líderes de la independencia colombiana, inauguró la educación en el nuevo país sudamericano. La historia comenzó a ser vista, desde el Estado, como la forma de legitimar y construir a la nación, lo que implicaba que impartirla en las aulas $y$ llevarla a las personas, era una imperante necesidad para ese proyecto.

Con el fin de ofrecer una mirada sobre las transformaciones de la enseñanza de la historia en Colombia, se realizó un breve balance historiográfico que indagó sobre la forma que tomó este tipo de educación en el país. Para ello, se recopiló información de diferentes fuentes, entre ellas, manuales, documentos oficiales y legislación, sumado a una docena de trabajos publicados que analizan la enseñanza de la historia y que dan cuenta de la producción bibliográfica sobre el tema en particular. A partir de dicha búsqueda, se realizó una contrastación y comparación de fuentes, para apreciar diferencias, tendencias, similitudes o transformaciones de la disciplina como objeto de enseñanza. Además, se tomaron como criterios de búsqueda las perspectivas teóricas, metodológicas y epistemológicas empleadas en los escritos educativos, como también su ubicación temporal, su enfoque disciplinar y su origen académico o político.

En dicho sentido, este estudio tiene como objetivo ofrecer una mirada panorámica al desarrollo y evolución de la enseñanza de la historia en el país, teniendo en cuenta los procesos de la vida nacional, las influencias educativas y políticas, y el significado de enseñar esta ciencia social en un país tan complejo como Colombia. Esto, acompañado de una reflexión, brindará herramientas para entender el presente: objetivo final de aprender del pasado.

\section{LA ENSEÑANZA DE LA HISTORIA \\ EN EL SIGLO XIX: UN PROCESO \\ DE CONSOLIDACIÓN NACIONAL}

El siglo decimonónico está lleno de procesos históricos que ameritan amplios análisis. En los territorios que ocupan la actual Colombia, los acontecimientos emancipadores frente a la metrópoli española, comenzaron a plantear distinciones en varios referentes de la vida política del nuevo país. La enseñanza o "instrucción pública", como se le conocía en aquella época, fue una de las primeras materias que el gobierno acogió en sus decretos y leyes para garantizar una "primera base del edificio social y sin la cual la República no es más que un vano nombre" (López, 1992).

Sin embargo, estos primeros decretos que oscilaban entre 1819 y 1821, no eran profundamente diferentes al modelo educativo colonial, ya que las nuevas instituciones educativas seguirían en manos de la Iglesia e impartirían saberes, también legados del pasado monárquico, tales como gramática castellana, latín, retórica y filosofía; uno de los aspectos que si cambió fue la enseñanza de política constitucional y de derechos de gentes, además, de manera sectorial se impartían clases de mineralogía, medicinas y algunos saberes liberales (López, 1992).

Durante la primera mitad de siglo, se intentó construir planes educativos que se desvanecían por su sentido utópico y por la carencia de educadores $y$ fondos económicos para suplir la naciente demanda. Respecto a ello, la historia no estaba explícitamente contenida en los programas; Miguel Aguilera, en su libro La enseñanza de la historia en Colombia, plantea que en 1826 se adoptó un plan de estudios:

... que abarcaba desde las escuelas de parroquia y cantón, hasta las facultades de carrera profesional. Incluyéndose en él la enseñanza de principios de geografía, cronología e historia, pero sin especificar la extensión de ninguna de estas asignaturas. En la Escuela de medicina se creó la clase de historia, pero de esta ciencia (Aguilera, 1951, p. 5).

Es de recordar que la historia era un asunto de interés de los sectores dirigentes del país, un ejemplo de ello fue la publicación de uno de los más insignes libros de historia de Colombia: Historia de la Revolución de la República de Colombia en la América Meridional que data de 1827 y escrito por uno de los más importantes políticos de aquel entonces, José Manuel Restrepo, una obra catalogada por el historiador German Colmenares (1989) como 
una "prisión historiográfica". Este concepto hace referencia a recordar que la historia era un asunto de interés de los sectores dirigentes del país, un ejemplo de ello fue la publicación de uno de los más insignes libros de historia de Colombia: Historia de la Revolución de la República de Colombia en la América Meridional que data de 1827 y escrito por uno de los más importantes políticos de aquel entonces, José Manuel Restrepo, una obra catalogada por el historiador German Colmenares como una "prisión historiográfica", concepto que engloba los lineamientos, a manera de apología, que ha poseído la escritura de la historia oficial (Colmenares, 1989).

En la segunda mitad del siglo xix, se dio el surgimiento de manuales de historia que comenzaron a ser utilizados para impartir su enseñanza. Uno de sus objetivos era construir el proyecto nacional que estaba mediado por el poder que tendría una escritura para legitimar las acciones de las elites colombianas. Luis Alarcón Meneses (2002) en su artículo "Construir la República y redefinir el pueblo, el discurso político en los manuales escolares colombianos del siglo xix", analiza las retóricas políticas que se empleaban en varios de los manuales escolares colombianos de aquella época, donde se reforzaban las propuestas enmarcadas en el republicanismo, que bajo figuras idealistas encarnadas por la ciudadanía, construían una participación novedosa para estas tierras colombianas.

Algunos historiadores decimonónicos emprendieron la labor de redactar vastos escritos que describieran los acontecimientos históricos del país. Varios de ellos fueron: Joaquín Acosta, quien escribió el Compendio histórico del descubrimiento y colonización de la Nueva Granada en el siglo decimosexto (1848); José Antonio de Plaza, autor de las Memorias para la historia de la Nueva Granada, desde su descubrimiento hasta el 20 de julio de 1810 (1850); José María Quijano Otero famoso por su Compendio de la historia patria (1870); Venancio Manrique, creador de los Rudimentos de Historia Universal para las escuelas de Colombia (1873); Constancio Franco con su Compendio de la Historia de la Revolución de Colombia.
Para el uso de las escuelas. Curso primero. La Independencia. 1810 a 1819 (1881; Juan Pablo Restrepo, autor del Compendio de Historia Patria (1891); José Joaquín Borda a quien se le atribuye el Compendio de historia de Colombia (1904) y para finalizar este recuento, Soledad Acosta de Samper y sus Lecciones de historia de Colombia (1908).

Respecto al estudio de los anteriores textos escolares, Silvia Juliana Rocha, en su monografía para optar por el título de historiadora: "La escritura de los manuales escolares de historia en Colombia durante la segunda mitad del siglo xix" (2008), analiza algunos de los anteriores manuales de historia, donde, utilizando las teorías de Norbert Lechner, propone que dichas "representaciones historiográficas", cumplieron con un papel y una función escenificadora y sacralizadora del pasado (Rocha, 2008 , p. 20). Las enmarca en un proyecto que pretendía construir una historia útil para moldear las mentalidades de aquellos que leyeran sus páginas. Normalmente estos textos tenían una periodización en común que, a partir de un orden cronológico, distinguía entre el descubrimiento, la conquista, la colonia y la república.

Además, sus fuentes se movían entre las "crónicas de Indias, relaciones de mando, documentación legal, memorias eclesiásticas, historias particulares, informes de expediciones, diccionarios, biografías, escritos políticos, sumas geográficas, manuscritos, cartas de viajeros, epistolarios, colecciones, periódicos y misceláneas" (Rocha, 2008, p. 21). Es importante señalar que no son abundantes los textos que trabajen este periodo en la enseñanza de la historia en Colombia, $y$ en ocasiones, el tema se aborda desde una perspectiva descriptiva que no ahonda en la vida de estos manuales dentro de la sociedad.

\section{EL SIGLO XX: DE LAS CLÁSICAS FORMAS DE ENSEÑAR HISTORIA HASTA LAS POSTURAS CONTEMPORÁNEAS}

Una de las obras que inauguró el siglo $x x$ en cuanto a los manuales de historia en Colombia fue la Historia de Colombia para la enseñanza secundaria de Jesús María Henao y Gerardo Arrubla, publicada en 1911, por una 
convocatoria del gobierno nacional. Esta obra tuvo una relación directa con la creación de la Academia Colombiana de Historia que fue fundada en 1902, y que para Miguel Aguilera, tuvo una fructífera y laudable labor, publicando gruesos volúmenes de narraciones, documentos, epistolarios, entre otros (Aguilera, 1951). La Historia de Colombia de este par de académicos, abogados de profesión, es un texto de orden positivista y patriótico, que se exacerbó por el candente momento que atravesaba el país, donde la integridad nacional había sido herida por la sesión de Panamá, además de un clima de posguerra civil, que tenía polarizada a la sociedad de ese momento (Tovar, 1999).

Ante la necesidad de reforzar el sentimiento nacional, la mencionada convocatoria buscaba la selección del texto que guiara la enseñanza de la historia patria en Colombia, a lo que Henao y Arrubla respondieron produciendo uno que poseía "claridad y método de la exposición, el respeto a la verdad histórica, la imparcialidad en relación con las agrupaciones partidistas y el santo amor a la Patria que buscaba inculcar" (Tovar, 1999, p. 2). Fue el mejor ejemplo, tal vez, de una enseñanza de la historia con un tono conservador, que fue leída con fervor las primeras décadas del siglo xx y que incluso hoy, sigue siendo consultada.

Para Colombia, la llegada de los gobiernos liberales en la primera mitad del siglo xx supuso un cambio en el orden tradicional que imperaba en el país -donde una especie de prolongación de la vida decimonónica del Estado regeneracionista de Rafael Núñez se apoderaba de las instituciones, los gobiernos y las gentes de la nación, esto a pesar de estar muy entrado el siglo xx-, una especie de anacronismo respecto al mundo. Por lo tanto, Alfonso López Pumarejo, un presidente liberal llegado el poder en 1934, adelantó un programa de gobierno que buscaba la modernización del país, adentrándose directamente en la construcción de un nuevo Estado.

Respecto a la enseñanza durante estos gobiernos, se le dio el carácter laico a la educación y se le entregó la responsabilidad al Estado de la instrucción del pueblo, arrebatándole a la Iglesia una función que tenía desde el periodo
Colonial. Adicionalmente, se promulgó la libertad de enseñanza y la gratuidad de la educación primaria en las instituciones educativas del Estado (Tirado, 1986).

Al respecto, la alfabetización y la lectura eran vistas como "una forma de redignificación de la vida, de acceso a la ciudadanía, de ampliación de horizontes, $y$ además una forma del conocimiento necesario que todo hombre debe tener del pasado de su sociedad $y$ de sus tradiciones" (Silva, 2002, p. 147). También la educación superior se vio favorecida con la modificación del sistema universitario, la reestructuración de la Universidad Nacional y la construcción de su campus central en Bogotá.

Uno de los historiadores que ha trabajado la educación durante este periodo es Renán Silva, quien desarrolla este tema principalmente en el artículo "Libros y lecturas durante la república liberal: Colombia, 1930-1946” (2002, pp. 141-169). Allí Silva recorre las formas de expansión de las redes culturales, motivadas por el Estado, con la intención de institucionalizar la ciudadanía y resaltar los derechos, más no los deberes, de la vida en la sociedad que se quería refundar. También analiza los procesos de alfabetización y expansión de los libros y la lectura en las diferentes regiones del país, dando cuenta así de los intentos - un poco fracasados-, por construir una nación sólida durante los gobiernos liberales entre 1930 y 1945. En el texto se exponen las formas para implementar una vida en torno a los ideales culturales del modernismo o de su proyecto "civilizatorio".

Este proceso alfabetizador nacional, emprendido principalmente por el gobierno de Alfonso López Pumarejo y su ministro de educación Luis López de Mesa, poseía proyectos para llevar a los pueblos o "aldeas", bibliotecas prácticas con textos y cartillas que fueran de fácil lectura para los campesinos y habitantes de los pequeños poblados del país. Este objetivo, según cuenta Silva, tuvo un relativo éxito, comparado con los inexistentes proyectos anteriores, por lo cual, gran parte del territorio

1 Este ideal fue defendido por Daniel Samper Ortega, director de la Biblioteca Nacional de Colombia entre 1931 y 1938. 
nacional se vio dotado de libros, con lugares de lectura en algunos casos inapropiados y con apoyos locales existentes pero escuetos. Las "Bibliotecas aldeanas" tuvieron ataques por parte de líderes conservadores que veían en ellas los ideales del partido liberal llevados a las masas populares.

Estas bibliotecas contenían libros principalmente para el desarrollo de saberes técnicos y prácticos, pero también estaban dotadas con libros catalogados como "información para la segunda enseñanza" o de "conocimientos generales" (Silva, 2002, p. 152). Dentro de estos últimos, que en su mayoría eran traídos del exterior y que eran llamados "cartillas", se encontraban: Historia de Grecia (C. A. Fyffe); Historia de Roma (C. Greighton); Economía Politica (W. S. Jevons) y Antigüedades Griegas (J. P. Mahaffy). Muchos de estos libros eran sugeridos para la preparación de los profesores (Silva, 2002).

Respecto a los avances en materia de enseñanza durante la República Liberal, existen varias tesis que, por un lado, plantean unos escuetos cambios en las políticas educativas, mientras que otros exponen verdaderas renovaciones desde el gobierno, en las materias mencionadas (Herrera, 1993). Un artículo de la profesora de la Universidad Pedagógica Nacional, Martha Herrera, titulado "La educación en la segunda República Liberal (1930-1946). Apuntes para una historiografía”, realiza un análisis de la producción historiográfica que versa sobre la enseñanza de la historia en Colombia, allí, plantea que una de las más importantes instituciones de este periodo fue la que resultó de la unión de varias corporaciones universitarias en la Escuela Normal Superior, donde se formaron gran parte de los académicos dedicados a las ciencias sociales que repercutirían en la segunda mitad de siglo xx en Colombia (Herrera, 1993).

La profesora Herrera sustenta dicha afirmación en un estudio elaborado por Jorge Orlando Melo titulado "La historia de la ciencia en Colombia", donde expone que "allí [en la Escuela Normal Superior] se formaron sistemáticamente, por primera vez en el país lingüistas, antropólogos, historiadores y otros científicos sociales(...) que, durante los cincuenta y los sesentas, contribuirían, en forma substancial a la consolidación de la Ciencia Social en Colombia" (Melo, 1986, p. 7).

Todo ello se complementa con la masiva inmigración de intelectuales que provenían de Europa, huyendo de las devastadoras consecuencias de los conflictos bélicos acaecidos en España o ad portas de explotar en todo el continente.

En contraste con dichos avances y coetáneo a ellos, el libro de Miguel Aguilera (1951), critica la estructura de la enseñanza que se brindaba entre 1937 y 1945, donde las reformas a algunos apartados del eje curricular, para el académico, eran un retroceso en la formación histórica del país. Según el autor, la historia que se ofrecía en los colegios intentaba "encarrilar la materia por la trocha arriesgada de la conciencia de clase" (p. 37), donde la cooperación, el trabajo colectivo y la sindicalización eran los temas ordenados desde el gobierno de Bogotá, para insertar en los programas escolares ideas Bolcheviques. "Temible ensayo, que duró poco tiempo, a Dios gracias (...)" (p. 47).

Esta obra de Aguilera es especial por su enfoque en la enseñanza, característica poco vista hasta ese momento (1951) en los escritos académicos de Colombia. Una parte importante del libro habla de la metodología de la historia, que para Aguilera debe centrarse en la exposición de las narraciones de los hechos, la descripción de personajes, las consecuencias de los acontecimientos y la delimitación de épocas, entre otras. Recomendaba treinta años de distancia entre los acontecimientos y su narración, para evitar "perturbar el ánimo", "(...) la Historia debe ser como el vino: necesita tiempo para que pase de la condición de mosto turbio y desagradable a la de jugo de contento y salud" (Aguilera, 1951, p. 30). Con ese periodo de tiempo, se intentaba eliminar las pasiones en la narración de los hechos y despolitizar la descripción de los acontecimientos que posiblemente, si fueran dichos en tiempos inmediatos o de reciente suceso, se enfocaría en la ideología de algún partido político.

Expresa también que el modelo para impartir historia debía seguir un orden sucesivo 
de acontecimientos: "por el aspecto lógico nada más natural que enseñar la Historia en orden estrictamente cronológico" (Aguilera, 1951, p. 33). Sin embargo, distingue entre los niveles de complejidad que deben tener los cursos de historia desde la primaria hasta la universidad. Es importante destacar que Aguilera veía a la memorización como una herramienta ineficiente, que debía entrar en desuso.

Respecto a la segunda mitad del siglo xx, un amplio sector historiográfico expone que el Bogotazo (concepto acuñado a una serie de revueltas violentas ocasionadas por el asesinato del candidato liberal a la presidencia Jorge Eliecer Gaitán), el 9 de abril de 1948, devolvió los tópicos de historia patria a las aulas. La ratificación de ver historia patria en los colegios, sumándole su intensificación, fue objeto del Decreto presidencial 2388, que buscaba cohesionar $y$ darle dignidad a lo nacional y lo ciudadano en el dividido país, buscando una formación de "hábitos democráticos, decoro personal y orgullo nacional a través de la historia" (Acevedo y Samacá, 2012, p. 225). Las clases estarían divididas en temáticas según la guía de la Academia Colombiana de Historia:

... causas internas y externas de la Independencia $y$ Guerra de Independencia; Gran Colombia; Nueva Granada y Confederación Granadina; Estados Unidos de Colombia y República de Colombia. Para el desarrollo de la cátedra, se consideró que cada periodo fuese tratado en diez clases a cargo de cinco o seis académicos en un lapso de ocho meses (Acevedo y Samacá, 2012, p. 225).

La década de los 50, tuvo una motivación en cuanto a los estudios que analizaban la educación en Colombia. Entre ellos se destacaron Las fronteras de los partidos en Colombia. Historia y comentarios de la legislación escolar en la República desde 1821 hasta el 13 de Junio de 1953, publicado ese último año y escrito por Julio Hoenisberg; "Historia de la educación en Colombia”, por José María Rodríguez Rojas, escrito entre 1954 y 1955; y Luis Bohórquez Casal, con su libro La evolución educativa en Colombia publicado en 1956.
Un interesante estudio que elabora Álvaro Acevedo Tarazona y Gabriel Samacá Alonso, es el titulado "La política educativa para la enseñanza de la historia de Colombia (19481990): de los planes de estudio por asignaturas a la integración de las ciencias sociales". En el artículo, se analizan con detenimiento los desarrollos de la enseñanza de la historia desde los organismos estatales, encargados de su reglamentación y contenido.

Sus análisis plantean que a partir de la década de los 60, la enseñanza de la historia comenzó a transformarse en el país, pasando de las temáticas con un enfoque patriótico a:

... aquella tendencia que quería darle al conocimiento social mayor impacto en la construcción de la democracia y el desarrollo. A partir de este momento se empezará a dar un lento distanciamiento de los postulados patrióticos para engranar el conocimiento histórico y geográfico en los paradigmas citados (Acevedo y Samacá, 2012, p. 227).

Para ello, el Ministerio de Educación Nacional promulgó un decreto en 1962 con el objetivo de incorporar "los programas analíticos de estudios sociales y filosofía para el primer y segundo ciclo de educación media". El contexto de la Guerra Fría promovía la democratización de la sociedad como barrera para la entrada del socialismo reinante en los países de Europa del Este y en Cuba, por ello, no es de extrañar que un decreto de este tipo emergiera en dicho momento histórico.

El contenido estipulado para las clases sería: "Prehistoria General, Americana y de Colombia", donde se formaría en el alumno "la conciencia de que la sangre y los sentimientos de los indios están vivos en nuestro pueblo y de cómo es indispensable, para el progreso de Colombia, comprender todas las tendencias y pasados que se fundan en nosotros" (Ministerio de Educación Nacional, 1963, pp. 14-15), sin embargo, la historia patria seguía presente en los currículos, respetando y manteniendo las instituciones de la República que habían poseído una "tradición civilista". En cuanto a metodología, el artículo de Acevedo y Samacá expone 
que acudían a propuestas un tanto novedosas que se remitían a la "observación del entorno inmediato, el acercamiento a los documentos históricos, la comparación entre el pasado y el presente o la utilización de material audiovisual se entrecruzaban con otras "tradicionales" como las "fechas-jalones" o el uso sistemático de las biografías" (2012, p. 231).

La próxima reforma sería en la década de los 80 , cuando se pensó en unificar las ciencias sociales, vinculando directamente otras disciplinas, especialmente a la Geografía. Este cambio estuvo precedido por unas recomendaciones que hicieron los historiadores Jaime Jaramillo Uribe y Jorge Orlando Melo en 1971, quienes redactaron para el Ministerio de Educación un texto que lleva por título "Claves para la enseñanza de la Historia”. Dicho escrito sugería una educación que tuviera como referentes los modernos usos historiográficos, mencionando a la Nueva Historia de Colombia como eje de las referencias curriculares. Los académicos resaltaban la necesidad de emplear una conceptualización y una utilización de ejemplos para potencializar la enseñanza y permitir que el estudiantado adquiera:

Un conocimiento integral de la Historia de Colombia, que comprenda sus instituciones sociales, políticas y culturales, a través de sus datos más significativos. El conocimiento histórico debe lograr que el estudiante aprenda a pensar los hechos de la Historia en sus conexiones hacia adentro y hacia fuera, es decir, a relacionar en sus influencias mutuas los hechos inmediatos que pertenecen a la propia realidad colombiana $y$ a éstos con los hechos exteriores que pertenecen a la Historia Universal directamente ligada a la nacional (Melo y Jaramillo, 1971, 1-2).

Este texto ofrece todo un esquema referencial para la enseñanza de la historia con una perspectiva interdisciplinar, que muestra a la historia como un objeto inacabado, complejo $y$ lleno de conocimientos básicos para la población estudiantil. Emplea un método de correlación de la Historia con la Geografía, donde ambas se enseñan simultáneamente, $y$ dependiendo de la edad, presenta ciclos intensivos separados por procesos históricos, donde los temas van desde la historia nacional, hacia la historia universal y las ciencias sociales. Además, el texto posee una valiosa guía bibliográfica para el desarrollo de los programas, donde se incluyen algunos de los trabajos académicos más novedosos y contributivos para ese momento.

Siguiendo con las reformas planteadas por el Ministerio, con la excusa de eliminar las barreras disciplinares e insertar la educación colombiana a los requerimientos del sistema globalizado, se propuso un proyecto integrado que reforzara las "actitudes democráticas", introduciendo también ideas de diversidades culturales para:

... desarrollar una conciencia sana de nacionalidad al tiempo que un aprecio por otras nacionalidades. Entender la identidad colombiana como una unidad que congrega la diversidad de formas de ser que coexisten en el país, que tienen su origen tanto en las diferencias regionales como en la presencia simultánea de lo tradicional y lo moderno (Abreu, 1988, pp. 12-16).

Según Acevedo y Samacá (2012), de las discusiones en torno a la elaboración de los marcos curriculares se priorizaron los esfuerzos por construir los métodos de enseñanza más que los contenidos, esto pretendía acercar las ciencias sociales a la "práctica y a la vivencia de valores democráticos" (p. 239), más "que a la acumulación de conocimientos" (p. 239). Todo ello con el fin de democratizar y exponer el desarrollo socioeconómico como "pilares fundamentales de este proyecto educativo al finalizar el siglo xx" (pp. 238-239).

En los primeros años del siglo xxi, aparecieron diversos textos que versan sobre la enseñanza de la historia en Colombia. Hacia el 2004 , se publicó un texto denominado Rutas pedagógicas de la historia, donde participan importantes historiadores de orden nacional como Mauricio Archila Neira, con un texto denominado "El historiador y la enseñanza de la historia"; Diana Obregón con "Rutas pedagógicas para el estudio de la historia en la 
educación básica en Bogotá”; o Bernardo Tovar Zambrano, quien escribió "La historia en el aula" (RED, 2004). Este último texto, destaca en especial la relación entre el aula, la investigación histórica y la enseñanza-aprendizaje de la historia.

Tovar argumenta que el aula es el "espacio de comunicación de lo producido en los campos de investigación", mientras que de la enseñanza describe su sujeción a los resultados de la academia y de un vaivén de políticas estatales, de normativas o de propósitos ideológicos, entre otros, que en muchas ocasiones se asemeja a una relación de verticalidad entre los conocimientos del docente "inmodificables" y las posibilidades memorísticas del estudiante, algo usual en los procesos de enseñanza aquí expuestos a lo largo de la historia del país. Para Tovar, debe existir una combinación de metodologías, que sin suprimir absolutamente la orientación anterior, plantee estrategias "que conduzcan a la comprensión histórica y con actividades cognoscitivas de elaboración propia de los estudiantes, es decir, prácticas de investigación, producción de conocimiento" (2004, p. 95).

El historiador propone que la narración "como una capacidad inherente a la mente humana" (Tovar, 2004, p. 95) que otorga significado al pasado y al presente, además de captar la atención y la curiosidad, debe ser empleada en la enseñanza de la historia. Además, gradualmente se deben propiciar los conocimientos con una perspectiva interdisciplinaria para buscar una visión global y comprehensiva. Este texto finaliza proponiendo convertir al aula en un taller de investigación.

Uno de los últimos libros que se ha editado con el objetivo de pensar la enseñanza es el que publicó la Universidad Pedagógica Tecnológica de Colombia en el 2011 y que lleva por título ¿Para qué enseñar historia? (Guerrero y Weisner, 2011), compilado por Javier Guerrero y Luis Weisner. Allí un valioso capítulo de Josep Fontana, presenta que la enseñanza del pasado expone a los poderosos, creando un ambiente de miedo entre ellos por la divulgación de su pasado. Por esta razón, se exhibe una manipulación y tergiversación de la historia, que a tomado usos comunes y corrientes.

También Fontana (Guerrero y Weisner, 2011) analiza la identidad y la relaciona con la memoria, lo que permite reconocerse así mismo; acude a las neurociencias para explicar que el cerebro almacena las experiencias anteriores y las reordena de manera organizada para procesar las nuevas vivencias, donde se produce como objeto final una conciencia. El llamado final del historiador español es el de utilizar el pasado para comprender el presente, trayendo análisis que aborden los fracasos de la historia para aprender de ellos y así alertar cuando exista evidencia de que se están recorriendo los mismos caminos que llevaron a la catástrofe, advierte que posiblemente en la actualidad se esté viviendo momentos de caos de los que ya se tiene suficiente. El escrito es un llamado de atención para la sociedad en general $y$ para los que hacen de las ciencias sociales su oficio cotidiano.

\section{¿PARA QUÉ ENSEÑAR HISTORIA? REFLEXIONES FINALES}

En la actualidad repensar la enseñanza de la historia, $y$ en general, la utilidad de las ciencias sociales $y$ humanas, requiere de una atención permanente, ya que es evidente el desprecio que en muchas ocasiones se percibe hacia esta en los discursos políticos y en las relaciones de poder. La historia todavía es vista como una recopilación arcaica de datos, que debe reposar en los anaqueles ocultos de las bibliotecas y que sus saberes no son prácticos para el mundo actual.

Allí es donde su enseñanza en las aulas de clase puede tomar un enorme valor. Mostrar a la historia como una ciencia viva, que no está limitada a lo ya escrito, y que puede ser reevaluada y puesta al servicio de la sociedad como una fuente de experiencia, es uno de los mayores retos de los que se dedican a su enseñanza e investigación. Vincular a la historia en los procesos humanos cotidianos, que les permitan a los individuos del común y a sus colectivos, repensar su pasado y combatir por ello, exige una responsabilidad ciudadana del profesorado $y$ de 
las personas historiadoras. Tal como lo plantea Carlos Pereyra (2005), la historia:

... más allá de la validez o legitimidad de los conocimientos que genera, acarrea consecuencias diversas para las confrontaciones y luchas del presente. No hay discurso histórico cuya eficacia sea puramente cognoscitiva; todo discurso histórico interviene (se inscribe) en una determinada realidad social donde es más o menos útil para las distintas fuerzas en pugna (pp. 12-13).

Esta función social era descrita por Marc Bloch, durante el transcurso de la Segunda Guerra Mundial, cuando decía:

No nos hemos atrevido a ser en la plaza pública la voz que clama en el desierto... hemos preferido encerrarnos en la quietud temerosa de nuestros talleres... De la mayor parte de nosotros se podrá decir que hemos sido unos buenos operarios. Pero, ¿hemos sido también buenos ciudadanos? (Fontana, 2003, p. 25).

La historia en las aulas debería ser aquella que, como Josep Fontana (2005) relata en su texto "Qué Historia enseñar", obedezca a la comprensión de los "problemas reales de los hombres y mujeres del pasado" (p. 18), una historia que sirva para educar, en lugar de adoctrinar, una historia reflexiva y crítica frente a las visiones egoístas donde los próceres son exaltados como héroes y sus proyectos son concebidos como irrefutables. Una enseñanza de la historia que permita a los individuos pensarse a sí mismos, pero también pensar a los otros, para encontrar similitudes o diferencias, donde la búsqueda por una identidad propia pase por reconocer la otredad, $y$ con ello evitar sectarismos $y$ visiones parciales de las sociedades.

El historiador especialista en la enseñanza de la historia Joan Pagés (2017), asegura que la enseñanza tiene dos partes fundamentales, necesaria una de la otra: la primera es el "saber", donde el conocimiento es esencial, y allí los saberes previos, mediados por una ardua formación en la producción de conocimiento -investigación-, posee una enorme importancia - difícil es enseñar lo que no se sabe-; $y$ la segunda es el "saber enseñar", espacio donde la pedagogía tiene un papel fundamental, que permite mediar entre un conocimiento y el estudiante, utilizando herramientas y dinámicas que revitalicen el ánimo por aprehender, según Pagés, el sentir aviva los caminos de la enseñanza. "Saber" y "saber enseñar" en su orden, deben mediar el proceso comprensivo de la enseñanza de la historia.

Es de resaltar que una de las labores más difíciles en Colombia es la de enseñar - y más aún si se está en un campo como el de las ciencias sociales y humanas que pueden llegar a ser vistas como inservibles, o peor aún, subversivas-. Las condiciones socioeconómicas del país cargan la labor docente con problemas estructurales que se ven intensificados cuando dicha acción se desarrolla en la Colombia profunda. Los docentes posiblemente pueden llegar a ser los únicos interlocutores entre aquellos sin voz y las sociedades. Es por ello que su labor se reviste de especial importancia y compromiso, donde no pueden ser aceptadas las posturas relajadas frente al conocimiento $y$ mucho menos frente a sus responsabilidades.

El personal docente debe dar herramientas útiles a sus estudiantes, que les permita indagar sobre las realidades actuales $y$ les ayude a comprender los acontecimientos que se viven actualmente, con una perspectiva histórica e integral: se debe enseñar a pensar históricamente, lo que permitirá tener una visión crítica y mediada por la duda, algo fundamental en una sociedad como la actual, donde el excesivo suministro indiscriminado de información está socavando la realidad de los hechos. Es por ello, que siguiendo las palabras de Bloch (1940) —uno de los padres de Annales - , quien vivió en un mundo en crisis, la enseñanza de la historia entre muchas otras tiene una función especial: "introducir 
un pellizco de conciencia en la mentalidad del estudiante. Ésta me parece que es la gran tarea que puede hacer quien enseña historia" (Fontana, 2003, p. 23).

\section{REFERENCIAS}

Abreu, M. (1988). Marco general de las ciencias sociales-Propuesta de programa curricular sexto grado de educación básica secundaria. Bogotá: Ministerio de Educación Nacional.

Acevedo, A. y Samacá, G. (2012). La política educativa para la enseñanza de la historia de Colombia (1948-1990): de los planes de estudio por asignaturas a la integración de las ciencias sociales. Revista Colombiana de Educación 62: 221-244. doi: http://dx.doi. org/10.17227/01203916.1637

Aguilera, M. (1951). La enseñanza de la Historia en Colombia. México: Instituto Panamericano de Geografía e Historia.

Alarcón, L. (2002). Construir la República y redefinir el pueblo. El discurso político en los manuales escolares colombianos del siglo xix. Historia Caribe 2, 7: 103-111.

Anderson, B. (1993). Comunidades Imaginadas. Reflexiones sobre el origen y la difusión del nacionalismo. México: Fondo de Cultura Económica.

Braudel, F. (1974). La Historia y las Ciencias Sociales. Barcelona: Alianza Editorial.

Colmenares, G. (1989). Las convenciones contra la cultura. Ensayos sobre la historiografía hispanoamericana del siglo XIX. Bogotá: Tercer mundo.

Corcuera de Mancera, S. (1997). Voces y silencios en la Historia: siglos XIX Y XXI. México: Fondo de Cultura Económica.

Febvre, L. (1970). Combates por la Historia. Madrid: Ariel.

Fontana, J. (2003). Qué Historia enseñar. Clío \& Asociados. La Historia Enseñada 7: 15-26.

Fukuyama, F. (1992). El fin de la Historia y el último hombre. Buenos Aires: Planeta.
Guerrero, J. y Weisner, L. (2011). ¿Para qué enseñar historia?. Bogotá: Universidad Pedagógica Tecnológica de Colombia, La Carreta.

Herrera, M. (1993). La educación en la segunda República Liberal (1930-1946). Apuntes para una historiografía. Revista colombiana de educación 26, 1-22.

Hobsbawm, E. (1983). Marxismo e historia social. México: Universidad Autónoma de Puebla.

López, L. (1992). "Santander y la educación: los colegios republicanos, una herencia perdurable". Revista Credencial Historia 28. Recuperado de http://www.banrepcultural. org/node/32310

Melo, J. (1986). La historia de la ciencia en Colombia. Rey, de la Universidad de Antioquia 203: 4-19.

Melo, J. y Jaramillo, J. (1971). Claves para la enseñanza de la Historia. Recuperado de http://www.jorgeorlandomelo.com/ calvespara.html

Moreno, M. (1999). La historia como arma y otros estudios sobre esclavos, ingenios y plantaciones. Barcelona: Crítica.

Pagés, J. (2017). ¿Qué ocurre en el mundo, qué historia enseñamos en la juventud a las escuelas? Reflexiones para adecuar la enseñanza de la historia a la realidad. XVIII Congreso colombiano de Historia, Medellín, Colombia.

Pereyra, C. (2005). Historia ¿Para qué? México: Siglo xxI.

RED. (2004). Rutas pedagógicas de la historia en la educación básica de Bogotá. Bogotá: Universidad Nacional, Alcaldía de Bogotá.

Rocha, S. (2008). La escritura de los manuales escolares de historia en Colombia durante la segunda mitad del siglo XIX (Monografía para optar por el título de historiadora).

Silva, R. (2002). Libros y lecturas durante la república liberal: Colombia, 1930-1946. Revista sociedad y economía 3, 141-169.

Spengler, O. (2014). La Decadencia de Occidente [1918-1923]. Madrid: Planeta. 
Tirado, A. (1986). La economía y lo social en la reforma constitucional de 1936. Lecturas de Economía 21, 81-98.

Tovar, B. (1999). "Jesús María Henao y Gerardo Arrubla: nueva lectura de una vieja Historia de Colombia”. Revista Credencial Historia 115. Recuperado de http://www. banrepcultural.org/node/32605
Tovar, B. (2004). Historia en el aula. Rutas pedagógicas de la historia en la educación básica de Bogotá. Bogotá: Universidad Nacional, Alcaldía de Bogotá.

Fecha de ingreso: 20/12/2017 Fecha de aprobación: 20/07/2018 